\title{
Relationship between acute high altitude response, cardiac function injury, and high altitude de-adaptation response after returning to lower altitude
}

\author{
Shengyue Yang ${ }^{1}$, Qiquan Zhou ${ }^{2,3 \#}$, Zifu Shi ${ }^{4}$, Enzhi Feng ${ }^{1}$, Ziqiang Yan $^{1}$, \\ Zhongxin $\operatorname{Tian}^{1}$, He Yin ${ }^{1}$, Yong Fan ${ }^{2,3}$ \\ ${ }^{1}$ Center of Respiratory Medicine, The 4th Hospital, Lanzhou Command, PLA, Xining, China \\ ${ }^{2}$ Department of High Altitude Diseases, College of High Altitude Military Medicine, Third Military Medical University, Chongqing, \\ China; ${ }^{\#}$ Corresponding Author: zhouqq9918@163.com \\ ${ }^{3}$ Key Laboratory of High Altitude Medicine of Ministry of Education and Key Laboratory of High Altitude Medicine of PLA, \\ Chongqing, China \\ ${ }^{4}$ The 68303 Troop Hospital of People's Liberation Army, Wuwei, China
}

Received 20 September 2013; revised 25 October 2013; accepted 8 November 2013

Copyright (C) 2013 Shengyue Yang et al. This is an open access article distributed under the Creative Commons Attribution License, which permits unrestricted use, distribution, and reproduction in any medium, provided the original work is properly cited. In accordance of the Creative Commons Attribution License all Copyrights (C) 2013 are reserved for SCIRP and the owner of the intellectual property Shengyue Yang et al. All Copyright (C) 2013 are guarded by law and by SCIRP as a guardian.

\section{ABSTRACT}

The relationship between acute high altitude response (AHAR), cardiac function injury, and high altitude de-adaptation response (HADAR) was assessed. Cardiac function indicators were assessed for 96 men (18 - 35 years old) deployed into a high altitude $(3700-4800 \mathrm{~m})$ environment requiring intense physical activity. The subjects were divided into 3 groups based on AHAR at high altitude: severe AHAR $(n=24)$, mild to moderate AHAR (Group $B, n=47$ ) and non-AHAR (Group C, 25); and based on HADAR: severe HADAR (Group $E, n=19$ ), mild to moderate HADAR (Group $F, n=40$ ) and non-HADAR (Group $G, n=37$ ) after return to lower altitude $(1,500 \mathrm{~m})$. Cardiac function indicators were measured after $\mathbf{5 0}$ days at high altitude and at $\mathbf{1 2}$ $h, 15$ days, and 30 days after return to lower altitude. Controls were 50 healthy volunteers (Group $D, n=\mathbf{5 0}$ ) at $\mathbf{1 5 0 0} \mathrm{m}$. Significant differences were observed in cardiac function indicators among groups A, B, C, and D. AHAR score was positively correlated with HADAR score $(r=0.863, P<0.001)$. Significant differences were also observed in cardiac function indicators among groups $\mathrm{D}, \mathrm{E}, \mathrm{F}$, and $\mathrm{G}, 12 \mathrm{~h}$ and

"The authors declare that no conflicts of interest exist.
15 days after return to lower altitude. There were no significant differences in cardiac function indicators among the groups, 30 days after return to lower altitude, compared to group $D$. The results indicated that the severity of HADAR is associated with the severity of AHAR and cardiac injury, and prolonged recovery.

Keywords: Acute High Altitude Response; Cardiac Function; Cardiac Structure; Myocardial Enzyme; Return to Lower Altitude; High Altitude De-Adaptation

\section{INTRODUCTION}

People sometimes live in high-altitude, hypoxic environments and this requires the body to make a complex series of compensatory changes in neurohumoral regulation, involving physiological, biochemical, and morphological changes to achieve a balance between the internal and external environment. When an individual returns to the normal oxygen environment, the body undergoes a series of corresponding changes resulting in the gradual elimination of the adaptive changes previously made. Many people undergoing this process experience symptoms such as dizziness, palpitations, chest tightness, drowsiness, precordial region pain, general fatigue, and weakness. This response is termed a high altitude 
de-adaptation the response, HADAR [1-5]. In severe cases, these symptoms can seriously affect the individual's work and life; due to the severity, some people have had to return to the high-altitude environment. HADAR is a problem of great concern and research.

Following the 2010 earthquake in Yushu, Qinghai Province, China, 96 young soldiers were rapidly deployed from a low altitude environment to a plateau area (elevation $3700-4800 \mathrm{~m}$ ) where they engaged in heavy physical labor for 50 days, before returning to their original station (elevation $1500 \mathrm{~m}$ ). This study was conducted to examine cardiac function, structure, and cardiac enzyme levels as part of an investigation into acute mountain sickness (acute high-altitude response; AHAR) as well as their symptoms at $12 \mathrm{~h}, 15 \mathrm{~d}$, and $30 \mathrm{~d}$ after their return to low altitude (HADAR response symptoms). This report describes the observed relationship among the severity of HADAR, AHAR, and heart damage.

\section{SUBJECTS AND METHODS}

\subsection{Subjects}

Following the Yushu earthquake, 96 male soldiers (18 - 35 years old; mean age $21.8 \pm 3.6$ years) were deployed to a high-altitude environment $(3700-4800 \mathrm{~m})$ to engage in heavy, manual labor. These men were the subjects of observations made once they reached the plateau and began participating in rescue operations (loading and unloading goods, cleaning up debris, etc.) involving heavy, physical labor for $10 \mathrm{~h}$ per day, every day. Following their high-altitude stay of $50 \mathrm{~d}$ and at $12 \mathrm{~h}, 15 \mathrm{~d}$, and $30 \mathrm{~d}$ after their return to their normal altitude environment $(1500 \mathrm{~m})$, their cardiac structure, function, and cardiac drive were examined. A control group (Group D) consisted of 50 soldiers who remained at the $1500 \mathrm{~m}$ altitude and engaged in a similar intensity of work. This control men, aged $19-20$ years old (mean age, $22.7 \pm$ 3.2 years) were similar in age, physical condition, work intensity to the group deployed to the plateau.

Based on AHAR symptom scores, the high-altitude group was divided into a severe AHAR group (Group A, $\mathrm{n}=24$ ), a mild to moderate group (Group $\mathrm{B}, \mathrm{n}=47$ ), and a asymptomatic group (Group $\mathrm{C}, \mathrm{n}=25$ ). Upon their return to the lower elevation, these same individuals were divided into another 3 groups depending on the severity of their HADAR symptoms: severe group (Group E, $\mathrm{n}=19$ ), mild to moderate group (Group $\mathrm{F}, \mathrm{n}=$ 40), and asymptomatic group (Group $\mathrm{G}, \mathrm{n}=37$ ). The study was approved by the Third Military Medical University medical ethics committee and the 68303 forces ethics committee; all subjects voluntarily participated in the observational part of the study and provided signed informed consent.

\subsection{Methods}

\subsubsection{AHAR Determination}

During the high-altitude observation, the subjects were observed daily by a physician and received an AHAR score, based on our AHAR symptom scoring system $[3,6]$. The subjects receiving a total of $1-4$ points were normal, 5 - 10 points were considered mildly symptomatic, 11 - 15 points were moderately symptomatic, and $>16$ points were considered severe. As a result of the scoring, 96 subjects were symptomatic for AHAR; 24 subjects were classified as severe (25.0\%, Group A), 47 subjects were mild to moderate (49.0\%, Group B). AHAR symptoms were not seen in 25 subjects $(26.0 \%$, Group C). To facilitate statistical analysis, subjects with mild or moderate AHAR symptoms were combined into one group (Figure 1).

\subsubsection{HADAR Determination}

Upon their return to low altitude, the Health Survey week congruent was used to score the HADAR symptoms among the test subjects (19). If the total number of points was $\leq 5$, the subjects were considered normal, 6 15 points were considered to be indicative of a mild reaction, 16 - 25 points were a moderate response, and $\geq 26$ points indicated a severe reaction. According to the HADAR scoring results, 59 (61.5\%) subjects were observed to have symptoms; 19 subjects were severe (Group E), and 40 subjects were mild to moderate (Group F). HADAR was not observed in 37 subjects (Group G) (Figure 1).

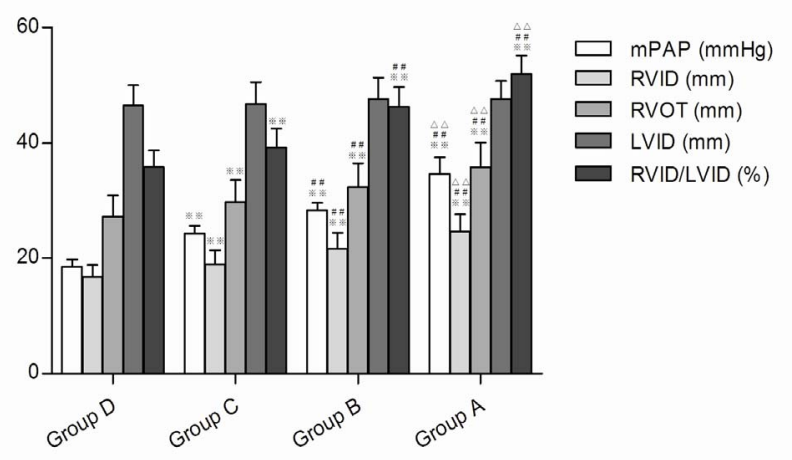

Figure 1. Comparisons of right cardiac structure, function, and mean pulmonary artery pressure among individuals at $3700 \mathrm{~m}$. mPAP: mean pulmonary arterial pressure; RVID: Right ventricular internal dimension; RVOT: Outflow tract of right ventricle; LVID: Left ventricular internal dimension. ${ }^{*} P<0.01$ vs Group D; ${ }^{\# \#} P<0.01$ vs Group C; ${ }^{\triangle \triangle} P<0.01$, vs Group B. 


\subsubsection{Cardiac Structure and Function Determination}

Color Doppler ultrasound (LOGIQ-type, GE Healthcare, Little Chalfont, UK) was used to detect the average pulmonary artery pressure (mPAP), right ventricular diameter (RVID), right ventricular outflow tract (RVOT), left ventricular diameter (LVID), left ventricular ejection fraction (LVEF), and myocardial performance index (Tei index). Inspections were made with the subject in the left lateral position, with measurement taken over a 1-h period with the subject in a resting state. The pulsed Doppler sample volume involved the measurement of the steady-state heart rate, the spectrum of the mitral and aortic blood flow, and the measurement of the peak A mitral valve flow between the end of the next cardiac cycle $\mathrm{E}$ peak time period (a line), and the aortic ejection time (b line). The Tei index is calculated according to the formula: Tei index $=(a b / b)$. Simpson's method was used to calculate each subject's LVEF [7]. mPAP measurements involved the determination of right ventricular ejection early time (RVPEP) and pulmonary blood flow acceleration time (AT), and was calculated according to the equation: $\mathrm{mPAP}(\mathrm{mmHg})=42.1(\mathrm{RVPEP} / \mathrm{AT})-15.7$ [8,9]. RVID, RVOT, and LVID were measured according to established methods.

\subsubsection{Determination of Cardiac Enzyme Levels}

A $3 \mathrm{ml}$, early morning, fasting blood sample was obtained, allowed to clot, and centrifuged at $4^{\circ} \mathrm{C}$ for $10 \mathrm{~min}$ (3500 rpm, centrifugal radius $=15 \mathrm{~cm})$. The resulting serum was collected and frozen at $-20^{\circ} \mathrm{C}$, until analyzed. The rate method was used to determine the concentrations of serum creatine kinase isoenzyme-MB (CK-MB) and lactate dehydrogenase isoenzyme-1 (LDH-1). Measurements were made using a kit purchased from Lanzhou Biochem Bio (lanzhou, China), in strict accordance with the manufacturer's instructions. An automatic biochemical analyzer (BS-400 type; Mindray Medical Instrumentation, Shenzhen, China) was used to take the measurements.

\subsection{Statistical Analysis}

SPSS 17.0 software (IBM, Armonk, NY, USA) was used for statistical analyses. Homogeneity of variance among the groups was determined by single factor analysis of variance and Tamhane variance analysis when heterogeneity of variance was observed. Differ- ences between 2 groups were compared using Student's t-test, and the results obtained at different times, within each group, were compared using a paired t-test. Corre- lation analysis was performed using the Pearson's linear correlation analysis. $P<0.05$ was considered statistically significant.

\section{RESULTS}

\subsection{Cardiac Structure, Function, and Myocardial Enzymes in Each Group}

The mPAP, RVID, RVOT, RVID/LVID ratio, Tei index, CK-MB level, and LDH-1 level were significantly higher in severe AHAR group than in mild to moderate AHAR group, asymptomatic group or control group (Figure 2). No significant differences were evident in the LVID values (Figure 1).

LVEF was significantly lower among severe AHAR group individuals than among those in mild to moderate AHAR group, asymptomatic group or control group. There was also a significant difference between mild to moderate AHAR group and asymptomatic group and control group; asymptomatic group was also significantly different from control group $(P<0.01)$ (Figure 2).

\subsection{Relationship between AHAR with HADAR}

Among the 96 subjects observed, 59 demonstrated HADAR symptoms upon returning to the lower altitude. Among the 24 subjects with severe AHAR, 18 experienced severe HADAR (75.0\%), 5 (20.8\%) experienced light to moderate HADAR, and $1(4.2 \%)$ did not experience HADAR symptoms. Of the 47 subjects who suffering from mild to moderate AHAR, 1 (2.1\%) experienced severe HADAR symptoms, 32 (68.1\%) experienced mild to moderate HADAR, and 14 (29.8\%) did not experience HADAR. Of the 25 subjects not suffering from AHAR, the HADAR was mild to moderate in 3 individuals and did not occur in $22(88.0 \%)$ subjects. A linear correlation analysis revealed that the total AHAR points and the

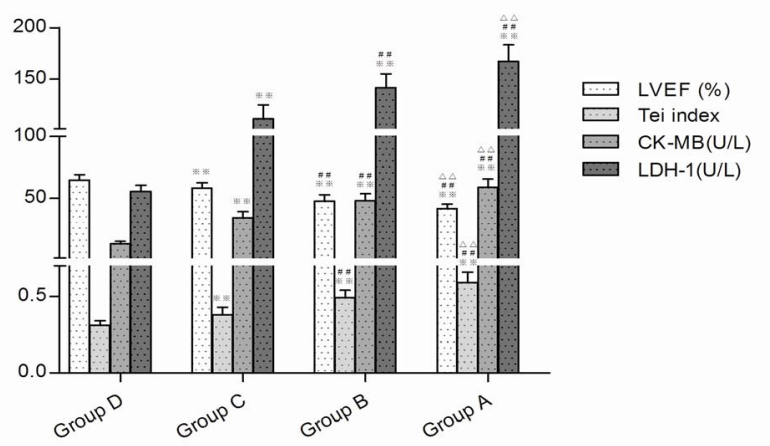

Figure 2. Comparisons of Tei index, left cardiac function, and cardiac muscle enzyme levels among groups at $3700 \mathrm{~m}$. LVEF: Left ventricular ejection fraction; Tei index: Cardiac muscle work index; CK-MB: Creatine kinase isoenzyme-MB; LDH1:Lactic dehydrogenase isoenzyme-1; Group A scores $\geq 16$; Group B scores 5 - 15; Group C scores $\leq 4$; Group D, normal controls at an altitude of $1500 \mathrm{~m}$. ${ }^{* *} P<0.01$ vs Group $\mathrm{D} ;{ }^{\# \#} P$ $<0.01$ vs Group C; ${ }^{\triangle \triangle} P<0.01$, vs Group B. 
total HADAR points exhibited a significant positive correlation $(\mathrm{r}=0.863, P<0.001)$.

\subsection{Relationship between HADAR Severity and Cardiac Structure, Function, and Cardiac Enzyme Levels after Returning to a Lower Altitude}

Upon returning to a lower altitude for $12 \mathrm{~h}$, Group $\mathrm{E}$ individuals demonstrated mPAP, RVID, RVOT, RVID/ LVID ratio, Tei index, CK-MB levels, and LDH-1 levels that were significantly higher than those for subjects in mild to moderate Group, asymptomatic group, and control group. LVEF was significantly lower in severe HADAR Group individuals than in individuals from mild to moderate HADAR Group, asymptomatic group, and control group; mild to moderate Group, asymptomatic group, and control group also demonstrated significant differences $(P<0.01)$. There were no significant differences in LVID between the groups (Figure 3).

After $15 \mathrm{~d}$ at low altitude, severe HADAR Group mPAP, RVID, RVOT, and RVID/LVID ratios were significantly higher than in mild to moderate HADAR Group, asymp- tomatic group, and control group $(P<$ $0.05)$; there was also a significant difference between mild to moderate HADAR Group individuals and those in asymptomatic group and control group $(P<0.05)$, but not between as- ymptomatic group and control group. The LVEF, Tei index, CK-MB levels, and LDH-1 levels in each group did not demonstrate a significant difference $(P>0.05)$. All data are shown in Figure 4.

After returning to a lower altitude for 30 days, there were no significant differences among the indicators for

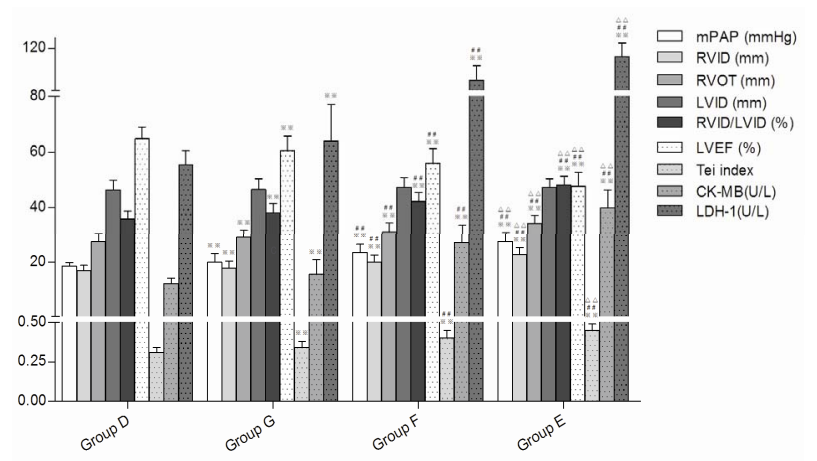

Figure 3. Relationship between HADAR, cardiac structure, function, and mean pulmonary artery pressures $12 \mathrm{~h}$ after returning to $1500 \mathrm{~m}$. mPAP: Mean pulmonary arterial pressure; RVID: Right ventricular internal dimension; RVOT: Outflow tract of right ventricle; LVID: Left ventricular internal dimension; LVEF: Left ventricular ejection fraction; Tei index: Cardiac muscle work index; CK-MB: Creatine kinase isoenzymeMB; LDH-1: Lactic dehydrogenase isoenzyme-1; Group E scores $\geq 26$; Group F scores 6 - 25; Group G scores $\leq 5$; Group $\mathrm{D}$, normal controls at an altitude of $1500 \mathrm{~m}$. ${ }^{* *} P<0.01$ vs Group D; ${ }^{\#} P<0.01$ vs Group G; ${ }^{\triangle \triangle} P<0.01$, vs Group F. any of the groups (Figure 5).

The diameter of pulmonary artery and the right ventricular outflow tract in the plateau at fiftieth days was significantly larger than the diameter of pulmonary artery and the right ventricular outflow tract in 30 days after returned to lower altitude, furthermore, compared with 50 days the exposure to high altitude, the diameter of right ventricular outflow tract was significantly larger than the diameter of pulmonary artery in 30 days after returned to lower altitude, suggesting that the right ventricular recovery than pulmonary artery slower recovery (Figure 6).

\section{DISCUSSION}

The results of this study showed that the right ventricular function, structure, myocardial enzyme level were significantly increased in the subjects with severe AHAR. In contrast, the left ventricular function was significantly lower. The responses of the human body to the high-altitude hypoxic environment and heavy physical work included more severe AHAR, pulmonary hypertension, changes in the right ventricle, reduced cardiac function and myocardial damage; but the changes in the left ventricular structure were less obvious. The reason may be that people engaged in heavy labor, in high-altitude, hypoxic environments, require a substantial increase in oxygen consumption. Therefore, when the body is exposed to a hypoxic environment, the hypoxia causes pulmonary vasoconstriction, pulmonary hypertension, and right ventricular load increase, leading to right ventricular enlargement. The AHAR severity, mPAP level,

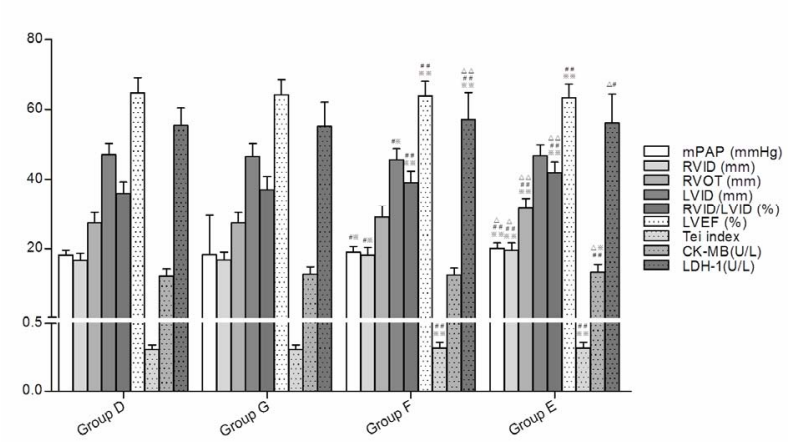

Figure 4. Relationship between HADAR, cardiac structure, function, and mean pulmonary artery pressure $15 \mathrm{~d}$ after returning to $1500 \mathrm{~m}$. mPAP: Mean pulmonary arterial pressure; RVID: Right ventricular internal dimension; RVOT: Outflow tract of right ventricle; LVID: Left ventricular internal dimension. LVEF: Left ventricular ejection fraction; Tei index: Cardiac muscle work index; CK-MB: Creatine kinase isoenzymeMB; LDH-1: Lactic dehydrogenase isoenzyme-1; Group E scores $\geq 26$; Group F scores 6 - 25; Group G scores $\leq 5$; Group $\mathrm{D}$, normal controls at an altitude of $1500 \mathrm{~m}$. ${ }^{* *} P<0.01$ vs Group D; ${ }^{\# \#} P<0.01$ vs Group G; ${ }^{\triangle \triangle} P<0.01$, vs Group F. 


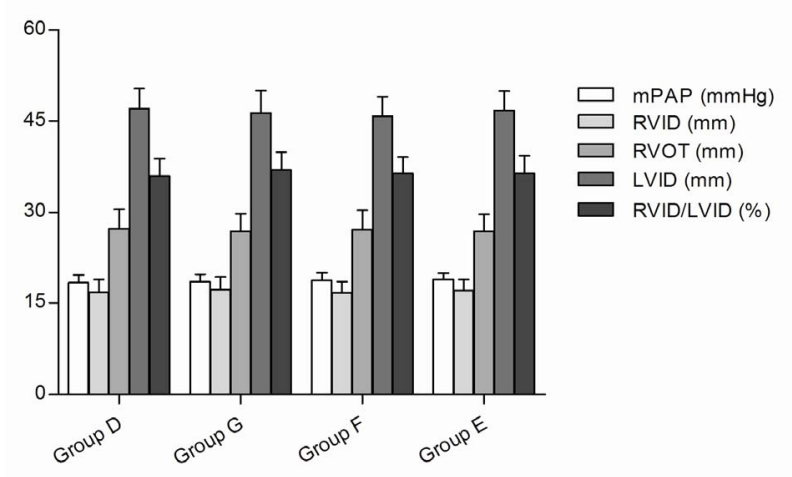

Figure 5. Relationship between HADAR and right cardiac structure, function, and mean pulmonary artery pressure $30 \mathrm{~d}$ after returning to $1500 \mathrm{~m}$. mPAP: Mean pulmonary arterial pressure; RVID: Right ventricular internal dimension; RVOT: Outflow tract of right ventricle; LVID: Left ventricular internal di- mension; Group E scores $\geq 26$; Group F scores 6 - 25; Group G scores $\leq 5$; Group D, normal controls at an altitude of $1500 \mathrm{~m}$.

and right ventricular size may be related to differences in individual tolerances and responses to hypoxia. In people with poor hypoxia tolerance, hypoxic mitochondrial ATP synthesis impairment in myocardial cells may result in a reduced myocardial energy supply, leading to obvious, severe symptoms. When hypoxia tolerance is relatively good, the inhibition of mitochondrial ATP synthesis is relatively mild, resulting in relatively mild heart damage and other symptoms [10-13]. For individuals who do not demonstrate AHAR, only sub-clinical effects on cardiac structure and function may occur.

The results of this study also show that the total AHAR and HADAR points showed a significant positive correlation. Upon returning to the lower altitude, subjects in the severe HADAR group demonstrated mPAP, RVID, RVOT, RVID/LVID ratio, Tei index, CK-MB level, and LDH-1 level results that were significantly higher than among those in the other groups; LVEF was significantly lower than in the other groups. Among subjects in the mild to moderate HADAR group there were also similar differences between these individuals and those in the asymptomatic and control groups. After returning to a lower elevation for $15 \mathrm{~d}$, individuals in the severe HADAR group continued to demonstrate mPAP, RVID, RVOT, and RVID/LVID ratio results that were significantly higher than those in the mild to moderate HADAR group, the asymptomatic HADAR group, or the control group. Subjects in the mild to moderate HADAR group also demonstrated significant differences in these parameters as compared with subjects in the asymptomatic HADAR group or in the control group. However, there were no differences between the HADAR group and the control group. The LVEF, Tei index, CK-MB level, and LDH-1 level values were restored to the values observed

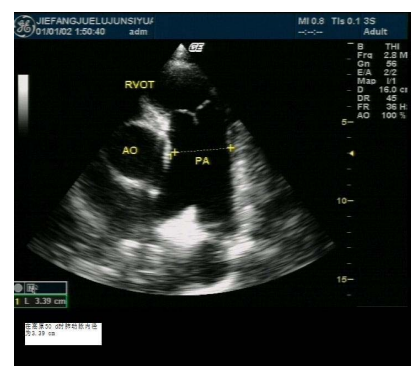

(a)

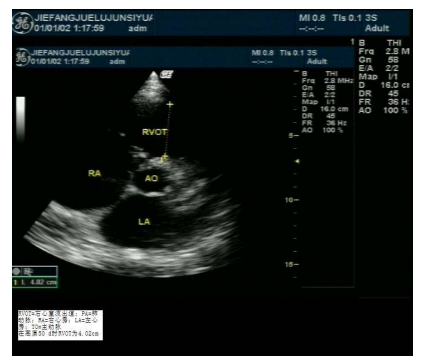

(c)

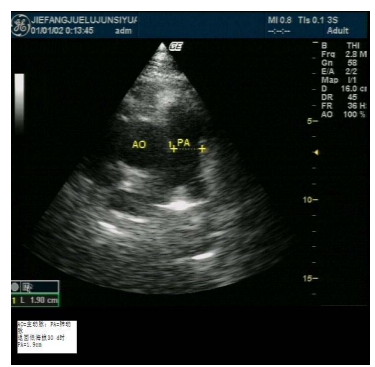

(b)

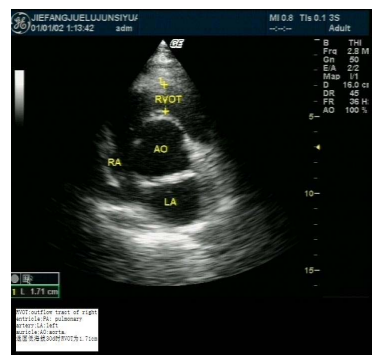

(d)
Figure 6. Pulmonary artery diameter and right ventricular outflow tract diameter return to lower altitude 30 days. (a) Pulmonary artery diameter up to $34 \mathrm{~mm}$ expsure 50 days under high altitude expansion environment; (b) Pulmonary artery diameter is $19 \mathrm{~mm}$ return to lower altitude 30 days; (c) Right ventricular outflow tract diameter up to $40 \mathrm{~mm}$ exposure 50 days under high altitude environment; (d) Right ventricular outflow tract diameter is $40 \mathrm{~mm}$ return to lower altitude 30 days.

in the control group level after being at the low altitude for 30 days,

The HADAR and AHAR severities were correlated with the degree of heart damage in these subjects. Those experiencing more severe AHAR while at the elevated elevation and having more significant HADAR symptoms upon their return to the low altitude experienced more severe structural damage to the right side of their hearts and a slower recovery time. After a period of adaptation to high-altitude, hypoxic environments, the return to lowaltitude environments results in hypoxia-reoxygenation injuries. The mechanism may involve the following components. 1) Energy metabolism: enhanced myocardial tissue hypoxia results in anaerobic glycolysis and decreased ATP generation, resulting in a decrease in cellular energy supply. Hypoxic damage to the mitochondria may result in the mitochondria not being effective when aerobic conditions are restored to the myocardial cells [9]. 2) Reactive oxygen species generation: oxygen free radicals (OFR) involved in clearing tissue hypoxia may result in the generation of reactive oxygen species; restoration of oxygen may result in the generation of a large number of OFR in the membranes of myocardial cells, resulting in the formation of lipid peroxides that react with intracellular proteins and nucleic acids. These peroxides may cause structural and func- 
tional changes to cells, leading to myocardial cell damage [14]. Zhang K, et al. [15] study confirmed, with enhanced of myocardial injuries, the increased levels of Malondialdehyde (MDA), lactate dehydrogenase (LDH) and interleukin 6 (IL-6), the LOB (Chrysoeriol7-O[-D-glucuronopyran-osyl-(1 $\rightarrow$ 2)-O-D-glucuronopyranoside]) can decreased plasma levels of MDA, LDH, IL-6, suggesting that the LOB could be a potential therapeutic agent for myocardial ischemia/reperfusion (I/R) injury and hypoxia/reoxygenation (H/R) injury. 3) Calcium overload: anaerobic glycolysis may enhance myocardial hypoxia, causing intracellular acidosis. As the extracellular $\mathrm{pH}$ gradually returns to normal after the restoration of normal oxygenation, intracellular and extracellular formation of transmembrane $\mathrm{pH}$ gradients may result in enhanced sodium and hydrogen exchange, increasing the intracellular $\mathrm{Na}$ concentration. Since the cells generate less ATP after reoxygenation, the cell membrane and sarcoplasmic reticulum calcium and sodium pump functions may be reduced, leading to intracellular calcium overload. The increase in the intracellular calcium concentration can further activate endothelial cells, promoting OFR generation, and leading to further damage [16,17]. But Li Q [18] study shows that endocannabinoids can suppresses calcium overload through inhibition of INCX during perfusion with simulated ischemic solution; the effects may be mediated by CB2 receptor via PTX-sensitive Gi/o proteins. 4) High altitude hypoxia stress induced myocardial injury, restore oxygen after myocardial injury has not been fully restored, or restore later than other functions. Hu J, et al. [19] study show that simple plateau hypoxia exposure endothelin (ET)-1 $\alpha$ concentrations gradually increased whereas HIF-1 expression in myocardial cells was significantly higher $(\mathrm{P}<$ $0.01)$. There was low pressure hypoxia exposure after myocardial mitochondria numbers were reduced during the initial phase of acute stress response to hypoxia and cellular injury but, later, mitochondrial numbers were restored to normal values. Plasma VEGF concentrations increased under exposure group hypoxia in low pressure hypoxia exposure, which were significantly higher than those of control group. Therefore $\mathrm{Hu}$ concluded that high-altitude hypoxia exposure: a) induced HIF-1 $\alpha$ expression; b) prompted adaptation/acclimatization after initial stress and cellular injury; and c) enhanced VEGF expression. The mechanism of HADAR and hypoxiareoxygenation injury on the body is extremely complex and requires further in-depth studies in order to more fully elucidate them.

\section{ACKNOWLEDGEMENTS}

The authors thank the personnel from the 68303 Infantry brigade and 68303 Troop Hospital of the People's Liberation Army for their assistance in this study. This work was funded by the National Science and
Technology Ministry (Grant\#2009BAI85B03) and Army Health Subject (Grant\# 2013BJZ032).

\section{AUTHOR CONTRIBUTIONS}

Conceived and designed the experiments: Shengyue Yang, Qiquan Zhou; Performed the experiments: Enzhi Feng, Ziqiang Yan, Zhongxin Tian, He Yin, Zifu Shi; Analyzed the data: Shengyue Yang; Contributed reagents/ materials/analysis tools: Zifu Shi; Wrote the manuscript: Shengyue Yang, Qiquan Zhou; English translation: Yong Fan.

\section{REFERENCES}

[1] Fan, Y. and Zhou, Q. (2012) Research progress of deadaptation to high altitude. Journal of Preventive Medicine of Chinese People's Liberation Army, 30, 227-230.

[2] He, B., Wang, J., Qian, G., Hu, M., Qu, X., Wei, Z., Li, J., Chen, Y., Chen, H., Zhou, Q. and Wang, G. (2013) Analysis of high-altitude de-acclimatization syndrome after exposure to high altitudes: A cluster-randomized controlled trial. PLoS One, 8, e62072.

http://dx.doi.org/10.1371/journal.pone.0062072

[3] Zhou, Q.Q., Yang, S.Y., Luo, Y.J., Qi, Y.S., Yan, Z.Q., Shi, Z.F. and Fan, Y. (2012) A randomly-controlled study on the cardiac function at the early stage of return to the plains after short-term exposure to high altitude. PLoS One, 7, e31097.

[4] Shi, Z., Zhou, Q., Xiang, L., Ma, S., Yan, C. and Luo, H. (2011) Three preparations of compound Chinese herbal medicines for de-adaptation to high altitude: A randomized, placebo-controlled trial. Journal of Chinese Integrative Medicine, 9, 395-401.

http://dx.doi.org/10.3736/jcim20110408

[5] Zhou, Q., Yang, S., Yuan, Z., Wang, Y., Zhang, X., Gao, W., Shi, Z., Yang, Y., Wu, Y., Fan, Y., Wang, G. and Gao, Y. (2012) A research in diagnostic criteria of high altitude de-adaptation for plateau migrants returning to the plains: a multicenter, randomized controlled trial. Medical Journal of Chinese People's Liberation Army, 37, 146-155.

[6] West, J.B. (2010) English translation of "Nomenclature, classification, and diagnostic criteria of high altitude disease in China". High Altitude Medicine \& Biology, 11, 169-172. http://dx.doi.org/10.1089/ham.2010.1014

[7] Simpson, J., Miller, O., Bell, A., Bellsham-Revell, H., McGhie, J. and Meijboom, F. (2012) Image orientation for three-dimensional echocardiography of congenital heart disease. The International Journal of Cardiovascular Imaging, 28, 743-753.

http://dx.doi.org/10.1007/s10554-011-9893-3

[8] Fakhri, A.A., Hughes-Doichev, R.A., Biederman, R.W. and Murali, S. (2012) Imaging in the evaluation of pulmonary artery hemodynamics and right ventricular structure and function. Heart Failure Clinics, 8, 353-372. http://dx.doi.org/10.1016/j.hfc.2012.04.004

[9] Zhao, S., Deng, Y.B., Chen, X.L. and Liu, R. (2012) As- 
sessment of right ventricular function in recipient twin of twin to twin transfusion syndrome with speckle tracking echocardiography. Ultrasound in Medicine and Biology, 38, 1502-1507.

http://dx.doi.org/10.1016/j.ultrasmedbio.2012.05.009

[10] Li, B., Liu, J. and Chen, L. (2005) Changes of adenylate content and distribution in myocardium and mitochondria of rats after hypoxic exposure. Medical Journal of $\mathrm{Na}$ tional Defending Forces In Northwest China, 26, 90-92.

[11] Li, J. and Xing, L. (2012) The effects of simulated 3500 $\mathrm{m}$ different hypoxic training on free radical metabolism and respiratory chain function of mitochondrial in myocardium after exhaustive running in rat. Journal of Shanghai Physical Education Institute, 36, 51-55.

[12] Rozova, K.V. (2008) Effect of normo-and hypobaric hypoxia on ultrastructure of the lung and myocardial tissue. Fiziolohichnyi Zhurnal, 54, 63-68.

[13] Zhao, Y. and Ao, H. (2011) Research progress of myocardial ischemia reperfusion injury. Chinese Circulation Journal, 26, 396-398.

[14] Kin, J.K., Pedram, A., Razandi, M. and Levin, E.R. (2006) Estrogen prevents cardiomyocyte apoptosis through inhibition of reactive oxygen species and differential regulation of p38 kinase isoforms. Journal of Biological Chemistry, 281, 6760-6767. http://dx.doi.org/10.1074/jbc.M511024200

[15] Zhang, K., Bai, Y., Song, T. and Zhang, G. (2013) In vivo and in vitro evidence of protective effects of a natural flavone on rat myocardial ischemia-reperfusion and hypoxia-reoxygenation injuries. Journal of Cardiovascular Pharmacology and Therapeutics, 18, 31-36. http://dx.doi.org/10.1177/1074248412461713

[16] Feygin, J., Hu, Q., Swingen, C. and Zhang, J. (2008) Relationships between regional myocardial wall stress and bioenergetics in hearts with left ventricular hypertrophy. American Journal of Physiology: Heart and Circulatory Physiology, 294, H2313-H2321. http://dx.doi.org/10.1152/ajpheart.01288.2007

[17] Zhang, D.W., Bian, Z.P., Xu, J.D., Wu, H.F., Gu, C.R., Zhou, B., Chen, X.J. and Yang, D. (2012) Astragaloside IV alleviates hypoxia/reoxygenation-induced neonatal rat cardiomyocyte injury via the protein kinase A pathway. Pharmacology, 90, 95-101. http://dx.doi.org/10.1159/000339476

[18] Li, Q., Cui, N., Du, Y., Ma, H. and Zhang, Y. (2013) Anandamide reduces intracellular $\mathrm{Ca}^{2+}$ concentration through suppression of $\mathrm{Na}^{+} / \mathrm{Ca}^{2+}$ exchanger current in rat cardiac myocytes. PLoS One, 8, e63386. http://dx.doi.org/10.1371/journal.pone.0063386

[19] Hu, J., Wang, Q.J., Hu, Y.H. and Li, Y.F. (2012) A study of high-altitude hypoxia-induced cell stress in murine model. Cell Biochemistry and Biophysics, 64, 85-88. http://dx.doi.org/10.1007/s12013-012-9374-x 